\title{
Interleukin 6 Targeting in Refractory Takayasu Arteritis: Serial Noninvasive Imaging is Mandatory to Monitor Efficacy
}

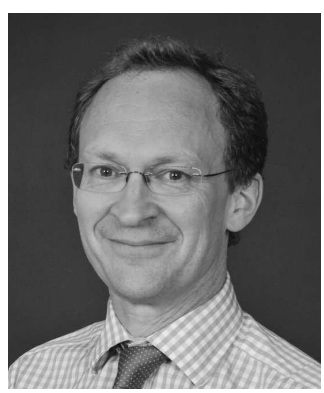

Takayasu arteritis (TA) is a true orphan disease. There are no published controlled clinical trials and the evidence on which to develop management strategies and therapeutic guidelines is rudimentary, particularly when compared to the advances seen in other vasculitides and inflammatory rheumatic diseases ${ }^{1}$. Additional significant challenges to more effective management exist. These include a lack of awareness of the disease and a resultant delay in diagnosis, as well as the absence of gold standards for the quantification of disease activity and imaging ${ }^{2}$. However, the outlook is beginning to change for the better, with early evidence pointing towards improved outcomes for patients ${ }^{3}$. This improvement reflects earlier use of combined immunosuppression and the increased availability of noninvasive imaging modalities, particularly ${ }^{18} \mathrm{~F}$-fluorodeoxyglucose positron emission computerized tomography $\left({ }^{18} \mathrm{~F}-\mathrm{FDG}-\mathrm{CT}-\mathrm{PET}\right)$, high-resolution ultrasound (US), magnetic resonance, and CT angiography (MRA and CTA).

Perhaps in light of the paucity of clinical trial evidence, corticosteroids remain the mainstay of therapy and achieve remission in $60 \%$ of cases ${ }^{4}$. However, when tapered, most patients relapse. Our practice is to initiate combination immunosuppressive therapy at diagnosis to optimize control of disease activity and steroid-sparing ${ }^{2}$. Small open-label studies support the efficacy of methotrexate, azathioprine, and mycophenolate ${ }^{5}$. Although cyclophosphamide is typically reserved for refractory or life-threatening disease, we have some concerns about its efficacy in TA, and its use is often contraindicated due to a need to preserve fertility in this young, predominantly female, patient group. Despite optimal combination immunosuppression, up to $30 \%$ of patients fail to respond adequately.

This observation led to the investigation of biologic therapies already available for other rheumatic diseases. Nearly a decade ago Hoffman and colleagues reported the initial open-label study demonstrating the efficacy of tumor necrosis factor- $\alpha$ (TNF- $\alpha$ ) blockade in $\mathrm{TA}^{6}$. Since then, these findings have been further validated in the most refractory of patients ${ }^{7,8}$. Akin to the experience in rheumatoid arthritis (RA), a proportion of patients with TA failed to respond to TNF- $\alpha$ antagonists, leading to the search for alternative therapeutic targets.

The biological actions of interleukin 6 (IL-6), alongside increased serum levels and its presence in the inflamed arterial wall in TA, suggest it plays a key pathogenic role $^{9,10}$. The TA inflammatory infiltrate comprises activated dendritic cells, $\alpha / \beta$ and $\gamma / \delta$ T lymphocytes, B lymphocytes, macrophages, and multinucleate giant cells. This inflammatory insult predisposes to intimal myofibroblast proliferation and fibrosis, with stenosis or occlusion of the arterial lumen (Figure 1). IL-6 is a pleiotropic cytokine, which may influence the function of many of these cell types. For example, IL-6 is important for T and B lymphocyte differentiation, generation of Th17 cells, stimulation of fibroblast proliferation, and for driving hepatocyte synthesis of acute phase proteins, including C-reactive protein (CRP) (Figure $1)^{11}$. Fortuitously, the humanized anti-IL-6 receptor monoclonal antibody tocilizumab and the anti-CD20 monoclonal antibody rituximab have been extensively studied in RA, and a preliminary report of 3 patients with TA treated with rituximab suggests B-cell depletion may be effective, although further confirmatory studies are required ${ }^{12}$. The first report of IL-6R blockade in TA described a dramatic prolonged response, with reduction in aortic wall thickness and resolution of ischemic symptoms, suggesting a disease-modifying effect ${ }^{13}$. Subsequently, small case series have described the further use of tocilizumab, with 44 patients included in a metaanalysis ${ }^{14}$. At time of analysis, just over half these patients continued taking tocilizumab therapy. Among the remainder, one-third achieved disease remission, and tocilizumab was stopped. In $40 \%$ of cases, treatment was withdrawn in the face of persistent radiological progression or disease relapse, while $20 \%$ suffered intolerable side effects ${ }^{14}$.

In this issue of The Journal, Tombetti and colleagues ${ }^{15}$

See Treatment of refractory TA with tocilizumab, page 2047

Personal non-commercial use only. The Journal of Rheumatology Copyright (c) 2013. All rights reserved. 


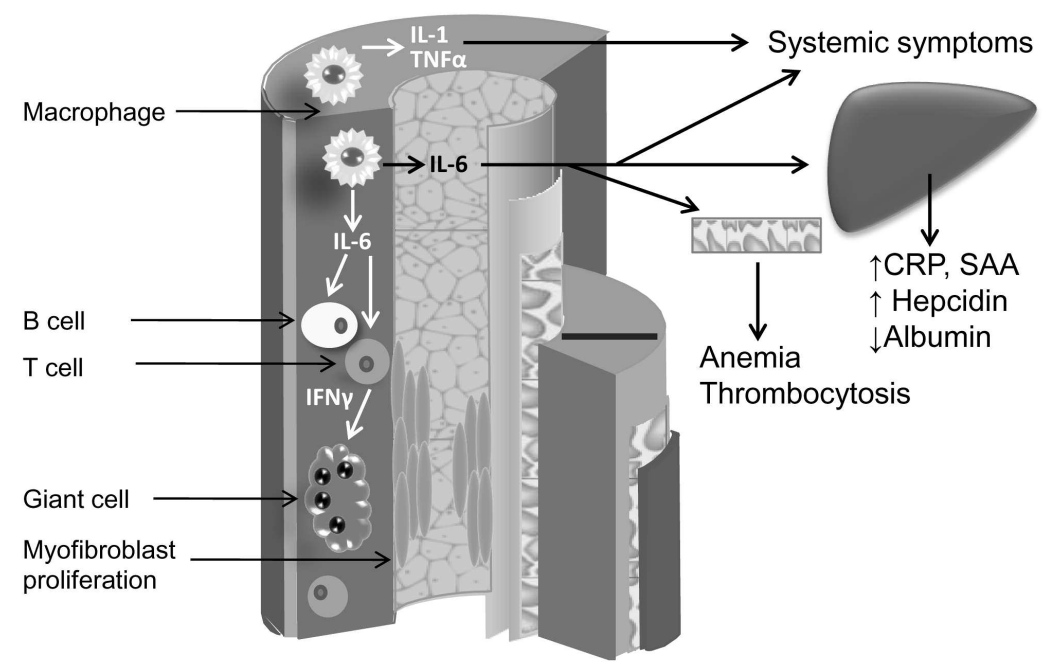

Figure 1. Interleukin 6 (IL-6) in Takayasu arteritis (TA). Serum levels and arterial wall expression of the proinflammatory cytokine IL- 6 are both increased in active TA. In the adventitia and the arterial wall IL-6 has the potential to drive T and B lymphocyte differentiation, the generation of Th17 cells, and to increase myofibroblast proliferation. Circulating IL-6 is responsible for inducing hepatocyte synthesis of acute phase proteins including C-reactive protein (CRP) and serum amyloid A (SAA), along with the systemic response and anemia of chronic disorder, thrombocytosis, and hypoalbuminemia. Although tocilizumab therapy will reverse these changes and lead to symptomatic improvement, this does not necessarily equate to resolution of arterial wall inflammation. Acute phase response proteins are no longer useful biomarkers and hence regular arterial imaging is required to identify responders and those with persistent progressive arterial disease.

report their retrospective review of 62 patients with TA seen in their center between 2004 and 2012. Of these, 29 (47\%) received biologic therapy for refractory disease, defined as failure to achieve a prednisone dose of $\leq 7.5 \mathrm{mg} / \mathrm{day}$, despite receiving at least 1 additional immunosuppressive drug. The percentage of patients requiring biologics is very high, with 22 patients receiving TNF- $\alpha$ antagonists and 7 receiving tocilizumab. This is the largest single series of patients treated with IL-6R blockade reported in detail.

The aim of the study ${ }^{15}$ was to evaluate the safety and efficacy of tocilizumab therapy in TA. Using a novel high resolution imaging approach the authors report that a proportion of patients responded well. However, their data also tells a cautionary tale, emphasizing the need for further realistic and thorough assessment of the benefits and limitations of IL6-R blockade in large vessel vasculitis. Although the study is small, retrospective, and lacks a control group, it raises important clinical issues. Critically, the authors stress that synthesis of acute phase proteins, such as CRP, serum amyloid A, and fibrinogen, will be suppressed in all patients receiving adequate doses of tocilizumab, and that the erythrocyte sedimentation rate (ESR) will also fall. Additionally, fever, malaise, and lethargy will resolve, while anemia, hypoalbuminemia, and thrombocytosis are typically reversed (Figure 1). This is true for all 7 patients in the current study, for the 44 included in the metaanalysis ${ }^{14}$, and in our clinical experience ${ }^{8}$. Thus, symptoms and biomarkers, such as CRP and ESR, cannot be relied upon as an estimate of disease activity, and decisions to change treatment should not be based on them. This includes dose reduction of immunosuppressants, which should be used in combination with tocilizumab, as in the current study. Further, the accuracy of disease activity indices, such as the US National Institutes of Health (NIH) criteria and Indian Takayasu Clinical Activity Score (ITAS) 20104,16, which incorporate ESR and/or CRP, may be compromised, a fact recognized by the authors ${ }^{15}$.

Although the response to IL-6R blockade supports a role for IL-6 in TA pathogenesis, the study findings also confirm that tocilizumab-induced suppression of symptoms and the acute phase response does not equate to complete inhibition of arterial wall inflammation and disease remission. This is further highlighted by reports of 3 other patients in whom tocilizumab therapy led to the rapid resolution of symptoms and the acute phase response, while disease in the arterial wall progressed, as evidenced by detection of new or progressive arterial injury using CTA and MRA ${ }^{17,18}$. Unfortunately, ${ }^{18} \mathrm{~F}$-FDG-CT-PET data are not available for the 7 patients included in the latest study. Of note, ${ }^{18}$ F-FDG-CT-PET scans performed pre- and postintroduction of tocilizumab seem to reveal complete suppression of FDG uptake ${ }^{14}$. Although these findings support a role for IL-6 in the increased FDG uptake, they also imply that ${ }^{18}$ F-FDG-CT-PET may be insensitive for detection of relapsing disease in those receiving tocilizumab. Nonetheless, ${ }^{18}$ F-FDG-CT-PET is not widely used during 
patient followup, due to limitations in availability, in sensitivity in partially-treated disease, and concerns regarding cumulative radiation dose.

Recognition of the comprehensive effect on symptoms and the inaccuracy of conventional biomarkers led the authors to recognize the critical importance of regular, scheduled, noninvasive imaging throughout the management of patients with TA treated with tocilizumab ${ }^{15}$. They used high resolution US to quantify changes in the common carotid and subclavian arteries, the abdominal aorta and femoral arteries. MRA analyzed the thoracoabdominal aorta and its major branches. Importantly, each arterial lesion was assessed individually. Complete treatment responders were those who had no progressive or new arterial lesions, no disease activity as measured by acute phase reactants and the NIH criteria, and a prednisone dose of $\leq 7.5 \mathrm{mg} /$ day for a minimum of 6 months. A patient was considered a partial responder if clinical improvement allowed the dose of prednisone to be reduced by $50 \%$. The strength of this study lies in the detailed imaging assessment of arterial lesions, which demonstrates the consistent improvement in sensitivity of MRA and US. The study results show that $3 / 7$ patients achieved a complete response. Imaging data revealed that 2 further patients had a single progressive arterial lesion, while the 2 remaining patients developed new lesions. Of the latter 4 patients, the authors chose to grade 1 as a partial responder, reflecting the reduction in prednisone dose by $50 \%{ }^{15}$. However, we would suggest that $3 / 7$ patients responded completely and that arterial disease progressed while taking treatment in 4/7.

The high quality imaging analysis included in the Tombetti, et al ${ }^{15}$ study also raises questions concerning the mechanisms underlying progressive stenosis despite treatment. Persistent low-grade inflammation is a likely cause. This conclusion is supported by the study of arterial specimens obtained during vascular surgery in patients with clinically inactive disease following conventional immunosuppression. Histological analysis revealed active arterial wall inflammation in $44 \%$ of cases ${ }^{4}$. In light of the complete suppression of IL-6 signaling by tocilizumab, other inflammatory pathways driven by B-cell activation and mediators, including TNF- $\alpha$, IL-17, and IL-18, are likely to be important. Additionally, a common arterial "response to injury" mechanism resulting in myofibroblast proliferation driven by growth factors with subsequent fibrosis, may predispose to stenosis. The location and degree of stenosis may also reflect anatomical and biomechanical changes secondary to injury. These include high shear stress exerted by arterial blood flow and stretch secondary to pulsation. Altered arterial anatomy may lead to regional differences in the activation of flow-response transcription factors including Kruppel-like factors 2 and 4, and the subsequent expression of vasoprotective and proinflammatory genes. Moreover, our understanding of IL-6 actions in the arterial wall is limited. Further consideration must be given to the suggestion that IL-6 may have a protective homeostatic role in the arterial wall. In giant cell arteritis, elevated IL-6 levels correlated with a reduced incidence of ischemic complications ${ }^{19}$. Thus, inhibition in some settings may predispose to arterial injury.

Inflammation and arterial injury in TA is a complex multifactorial process. Inhibition of IL-6 signaling may provide another therapeutic tool for some patients with refractory TA, with detailed noninvasive imaging mandatory during followup, due to the complete suppression of the acute phase response. The importance of high-resolution US and MRA for the detection of subclinical progressive disease in those receiving tocilizumab is shown in the current study. Further advances may allow us to discern the nature of these lesions and distinguish inflammatory change from arterial remodelling or chronic fibrotic disease. The development of novel PET ligands may be useful in this regard ${ }^{20}$. Combination imaging data of this type would allow stratified therapy, with optimized regimens developed and selected specifically for inflammatory lesions. In contrast, myofibroblast proliferation might be targeted more appropriately using drugs with an anti-proliferative action, such as sirolimus and everolimus. Nonetheless, the perennial challenge in this orphan disease remains, namely, the need to initiate prospective, multinational controlled clinical trials, with defined end-points incorporating novel disease activity indices, such as ITAS2010 and noninvasive image scores.

\author{
TARYN YOUNGSTEIN, BSc, MRCP, \\ Specialist Registrar in Rheumatology; \\ JUSTIN C. MASON, PhD, FRCP, \\ Professor of Vascular Rheumatology. \\ Rheumatology Section and Vascular Sciences, \\ Faculty of Medicine, Imperial College London, \\ Hammersmith Hospital, Du Cane Road, \\ London W12 0HS, UK
}

Supported in part by the Imperial College London National Institute for Health Research Biomedical Research Centre funding scheme.

Address correspondence to Professor Mason; Vascular Sciences, Imperial College London, Hammersmith Hospital, Du Cane Road, London, W12 0NN, UK. E-mail: justin.mason@imperial.ac.uk

\section{REFERENCES}

1. Mukhtyar C, Guillevin L, Cid MC, Dasgupta B, de Groot K, Gross $\mathrm{W}$, et al. EULAR recommendations for the management of large vessel vasculitis. Ann Rheum Dis 2009;68:318-23.

2. Mason JC. Takayasu arteritis - advances in diagnosis and management. Nat Rev Rheumatol 2010;6:406-15.

3. Ohigashi H, Haraguchi G, Konishi M, Tezuka D, Kamiishi T, Ishihara T, et al. Improved prognosis of Takayasu arteritis over the past decade-comprehensive analysis of 106 patients. Circ J 2012;76:1004-11.

4. Kerr GS, Hallahan CW, Giordano J, Leavitt RY, Fauci AS, Rottem M, et al. Takayasu arteritis. Ann Intern Med 1994;120:919-29.

5. Liang P, Hoffman GS. Advances in the medical and surgical Personal non-commercial use only. The Journal of Rheumatology Copyright @ 2013. All rights reserved. 
treatment of Takayasu arteritis. Curr Opin Rheumatol 2005;17:16-24.

6. Hoffman GS, Merkel PA, Brasington RD, Lenschow DJ, Liang P. Anti-tumor necrosis factor therapy in patients with difficult to treat Takayasu arteritis. Arthritis Rheum 2004;50:2296-304.

7. Comarmond C, Plaisier E, Dahan K, Mirault T, Emmerich J, Amoura Z, et al. Anti TNF- $\alpha$ in refractory Takayasu's arteritis: Cases series and review of the literature. Autoimmun Rev 2012;11:678-84.

8. Youngstein T, Peters JE, Hamdulay SS, Mewar D, Price-Forbes A, Lloyd M, et al. Serial analysis of clinical and imaging indices reveals prolonged efficacy of TNF- $\alpha$ and IL- 6 receptor targeted therapies in refractory Takayasu arteritis. Clin Exp Rheumatol 2013 Sep 30 [Epub ahead of print].

9. Noris M, Daina E, Gamba S, Bonazzola S, Remuzzi G. Interleukin-6 and RANTES in Takayasu arteritis: a guide for therapeutic decisions? Circulation 1999;100:55-60.

10. Park MC, Lee SW, Park YB, Lee SK. Serum cytokine profiles and their correlations with disease activity in Takayasu's arteritis. Rheumatology 2006;45:545-8.

11. Nishimoto N, Kishimoto T. Interleukin 6: from bench to bedside. Nat Clin Pract Rheumatol 2006;2:619-26.

12. Hoyer BF, Mumtaz IM, Loddenkemper K, Bruns A, Sengler C Hermann KG, et al. Takayasu arteritis is characterised by disturbances of B cell homeostasis and responds to B cell depletion therapy with rituximab. Ann Rheum Dis 2012;71:75-9.

13. Nishimoto N, Nakahara H, Yoshio-Hoshino N, Mima T. Successful treatment of a patient with Takayasu arteritis using a humanized anti-interleukin-6 receptor antibody. Arthritis Rheum 2008; 58:1197-2000.
14. Abisror N, Mekinian A, Lavigne C, Vandenhende MA, Soussan M, Fain O. Tocilizumab in refractory Takayasu arteritis: A case series and updated literature review. Autoimmun Rev 2013;12:1143-9.

15. Tombetti E, Franchini S, Papa M, Sabbadini MG, Baldissera E. Treatment of refractory Takayasu arteritis with tocilizumab: 7 Italian patients from a single referral center. J Rheumatol 2013;40:2047-51.

16. Misra R, Danda D, Rajappa SM, Ghosh A, Gupta R, Mahendranath $\mathrm{KM}$, et al. Development and initial validation of the Indian Takayasu Clinical Activity Score (ITAS2010). Rheumatology 2013;52:1795-801.

17. Bredemeier M, Rocha CM, Barbosa MV, Pitrez EH. One-year clinical and radiological evolution of a patient with refractory Takayasu's arteritis under treatment with tocilizumab. Clin Exp Rheumatol 2012;30:S98-100.

18. Xenitidis T, Horger M, Zeh G, Kanz L, Henes JC. Sustained inflammation of the aortic wall despite tocilizumab treatment in two cases of Takayasu arteritis. Rheumatology 2013;52:1729-31.

19. Hernandez-Rodriguez J, Segarra M, Vilardell C, Sanchez M, Garcia-Martinez A, Esteban MJ, et al. Elevated production of interleukin-6 is associated with a lower incidence of disease-related ischemic events in patients with giant-cell arteritis: angiogenic activity of interleukin- 6 as a potential protective mechanism. Circulation 2003;107:2428-34.

20. Pugliese F, Gaemperli O, Kinderlerer AR, Lamare F, Rimoldi OE, Mason JC, et al. Imaging of vascular inflammation with [11C]-PK11195 and PET/CT angiography. J Am Coll Cardiol 2010;56:33-9.

J Rheumatol 2013;40:1941-4; doi:10.3899/jrheum.131232 